\title{
Valence bond phases of herbertsmithite and related copper kagome materials
}

\author{
M. R. Norman $\odot,{ }^{1}$ N. J. Laurita, ${ }^{2,3}$ and D. Hsieh ${ }^{2,3}$ \\ ${ }^{1}$ Materials Science Division, Argonne National Laboratory, Argonne, Illinois 60439, USA \\ ${ }^{2}$ Department of Physics, California Institute of Technology, Pasadena, California 91125, USA \\ ${ }^{3}$ Institute for Quantum Information and Matter, California Institute of Technology, Pasadena, California 91125, USA
}

(Received 30 October 2019; revised manuscript received 20 December 2019; published 16 January 2020)

\begin{abstract}
Recent evidence from magnetic torque, electron spin resonance, and second harmonic generation indicate that the prototypical quantum spin liquid candidate, herbertsmithite, has a symmetry lower than its x-ray refined trigonal space group. Here we consider known and possible distortions of this mineral class, along with related copper kagome oxides and fluorides, relate these to possible valence bond patterns, and comment on their relevance to the physics of these interesting materials.
\end{abstract}

DOI: 10.1103/PhysRevResearch.2.013055

The nature of the ground state of the nearest-neighbor antiferromagnetic Heisenberg model on a kagome lattice (KAHM) has proven to be a challenging problem. Numerical simulations indicate that a variety of different states have comparable energies, including gapped spin liquids, gapless spin liquids, and valence bond solids. This is reflected in the energy spectrum of clusters from exact diagonalization studies, which shows a dense array of excited states extending down to zero energy [1]. In real materials, further richness emerges due to the presence of anisotropic interactions, such as Dzyaloshinskii-Moriya, as well as longer-range exchange. In this context, the lack of observation of an ordered ground state down to $20 \mathrm{mK}$ in herbertsmithite, $\mathrm{ZnCu}_{3}(\mathrm{OH})_{6} \mathrm{Cl}_{2}$, a mineral where copper ions sit on a perfect kagome lattice, has been a significant result [2-4].

In reality, though, herbertsmithite is far from perfect. Single crystals typically contain $15 \%$ of copper ions sitting on interlayer sites nominally occupied by zinc [5]. Moreover, despite $\mathrm{x}$-ray refinements of the crystal structure which indicate perfect trigonal symmetry $(\mathrm{R} \overline{3} \mathrm{~m})$, magnetic torque and electron spin resonance [6] find a breaking of the threefold trigonal axis. This has been recently amplified by secondharmonic-generation (SHG) data, which is consistent with a monoclinic space group that breaks inversion [7].

To put these results in context, it is first helpful to review known distortions in this mineral class, as well as related materials. The $\mathrm{Cu}_{4}(\mathrm{OH})_{6} \mathrm{Cl}_{2}$ polymorph from which herbertsmithite arises via $\mathrm{Zn}$ substitution for $\mathrm{Cu}, \mathrm{Zn}_{x} \mathrm{Cu}_{4-x}(\mathrm{OH})_{6} \mathrm{Cl}_{2}$, is clinoatacamite with a monoclinic $\mathrm{P} 2_{1} / \mathrm{n}$ space group [8]. On $\mathrm{Zn}$ doping, an intermediate $\mathrm{R} \overline{3}$ phase (Zn-paratacamite) is stabilized between $R \overline{3} \mathrm{~m}$ at high temperatures and $\mathrm{P} 2_{1} / \mathrm{n}$

Published by the American Physical Society under the terms of the Creative Commons Attribution 4.0 International license. Further distribution of this work must maintain attribution to the author(s) and the published article's title, journal citation, and DOI. at low temperatures. Eventually, the $\mathrm{P} 2_{1} / \mathrm{n}$ phase disappears, and then for $x$ beyond about 0.34 , so does the R $\overline{3}$ phase [9]. For Mg-paratacamite, the $\mathrm{R} \overline{3}$ phase has been detected up to $x=0.62$ [10]. To understand the nature of these two structural phases, we employ the crystallographic tools AMPLIMODES [11] and ISODISTORT [12].

The $\mathrm{R} \overline{3}$ phase is driven by an $\mathrm{F}_{2}^{+}$distortion mode resulting in a quadrupling of the unit cell in the planar directions [Fig. 1 (left)]. Here $\mathrm{F}$ is equal to $\left(0, \frac{1}{2}, 1\right)$ in hexagonal reciprocal lattice units and is related to the $M\left(\frac{1}{2}, 0,0\right)$ point of the hexagonal zone (the difference from $\mathrm{M}$ reflects the $\mathrm{ABC}$ stacking of layers in the rhombohedral lattice). The resulting crystallographic distortion from the $\mathrm{F}_{2}^{+}$mode is shown in Fig. 2. Basically, the interlayer sites (which would nominally be occupied by $\mathrm{Zn}$ in stoichiometric herbertsmithite) divide into two sets, one showing octahedral coordination ( $1 / 4$ of these sites) and the other a Jahn-Teller distorted $2+2+2$ coordination (the remainder). Around the first type, the atoms on the kagome plane rotate about it. This is known as a polar vortex [15] (more formally, an axial toroidal dipole [16]). The distortion pattern around the other interlayer sites has aspects of this as well but is more complicated. The actual crystal structure is even more complicated, given the presence of $\mathrm{F}_{1}^{+}$and $\Gamma_{2}^{+}$ secondary modes [Fig. 1 (left)]. Looking at just the copper kagome sites [17], one finds two crystallographically distinct sites. This leads to a distribution of $\mathrm{Cu}-\mathrm{Cu}$ kagome distances. The strongest singlet bond (largest $\mathrm{Cu}-\mathrm{O}-\mathrm{Cu}$ bond angle, the superexchange scaling with the bond angle [18]) forms a pinwheel pattern, as shown in Fig. 3 (left). This same pattern is seen in copper kagome fluorides such as $\mathrm{Rb}_{2} \mathrm{Cu}_{3} \mathrm{SnF}_{12}$ [19]. Such a pinwheel valence bond pattern has been previously discussed in the KAHM literature given its favorable energetics [20]. Note that this phase differs subtly from the so-called diamond valence bond solid, as resonances around diamonds would take one outside of the ground-state manifold [21] since those other bonds are not equivalent to the strong bonds. That is, because of the lattice distortion, there is an exchange energy cost for a diamond resonance that can be estimated as $2\left(2 J_{12}-J_{11}-J_{22}\right)$, where $J_{12}$ is the superexchange of the strong bond and 1 and 2 refer to the two crystallographically 

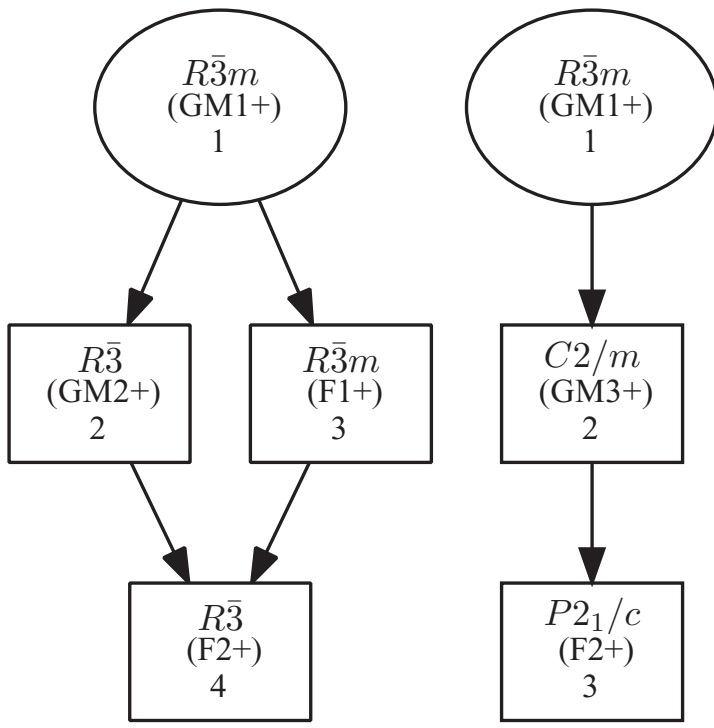

FIG. 1. Group-subgroup relation leading to (left) $\mathrm{R} \overline{3}$ and (right) $\mathrm{P} 22_{1} / \mathrm{c}$ (equivalent to $\mathrm{P} 2_{1} / \mathrm{n}$ ) [13]. As indicated in these graphs, the primary distortion mode is $\mathrm{F}_{2}^{+}$, and the secondary modes, arising from the intermediate groups, are $\Gamma_{2}^{+}$and $\mathrm{F}_{1}^{+}$for $\mathrm{R} \overline{3}$ and $\Gamma_{3}^{+}$for $\mathrm{P} 2{ }_{1} / \mathrm{c}$.

distinct kagome sites. For a resonance around a pinwheel, the energy cost is $6\left(J_{12}-J_{12}^{\prime}\right)$, where $J_{12}^{\prime}$ refers to the $1-2$ bond with the smaller $\mathrm{Cu}-\mathrm{O}-\mathrm{Cu}$ bond angle. Given the linear relation of $J$ with the $\mathrm{Cu}-\mathrm{O}-\mathrm{Cu}$ bond angle for the bond angle range appropriate to these materials $[18,22,23]$, the estimated cost of a diamond resonance is $0.54 J_{12}$ and a pinwheel resonance $1.45 J_{12}$ for $x=0.29$ [9]. These differences become much smaller as $x$ increases (for Mg-paratacamite at $x=0.62$, they become $0.03 J_{12}$ and $0.02 J_{12}$, respectively [10]).

The related $\mathrm{P} 2{ }_{1} / \mathrm{n}$ phase seen in clinoatacamite also arises from an $\mathrm{F}_{2}^{+}$distortion mode, the difference being due to different secondary modes $\left[\Gamma_{3}^{+}\right.$only for $\mathrm{P} 2_{1} / \mathrm{n}$; Fig. 1 (right)]. The change in the overall distortion pattern leads to the strong bond now having a herringbone-like structure [Fig. 3 (right)].

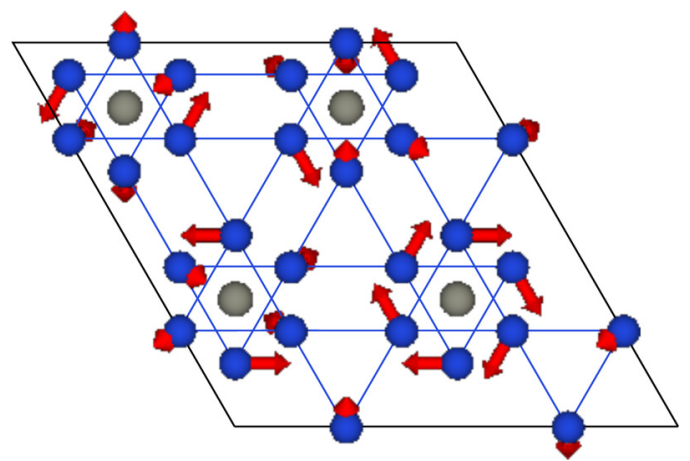

FIG. 2. $\mathrm{F}_{2}^{+}$distortion mode for the $\mathrm{R} \overline{3}$ phase of Zn-paratacamite [9] from AMPLIMODES [11] plotted using VESTA [14]. Only the copper/zinc ions are shown in an intersite plane and the two kagome planes that sandwich it (blue kagome, gray intersite). Note the vortexlike motion of the kagome coppers about one of the intersites. This occurs as well for the oxygen, hydrogen, and chlorine ions (not shown).

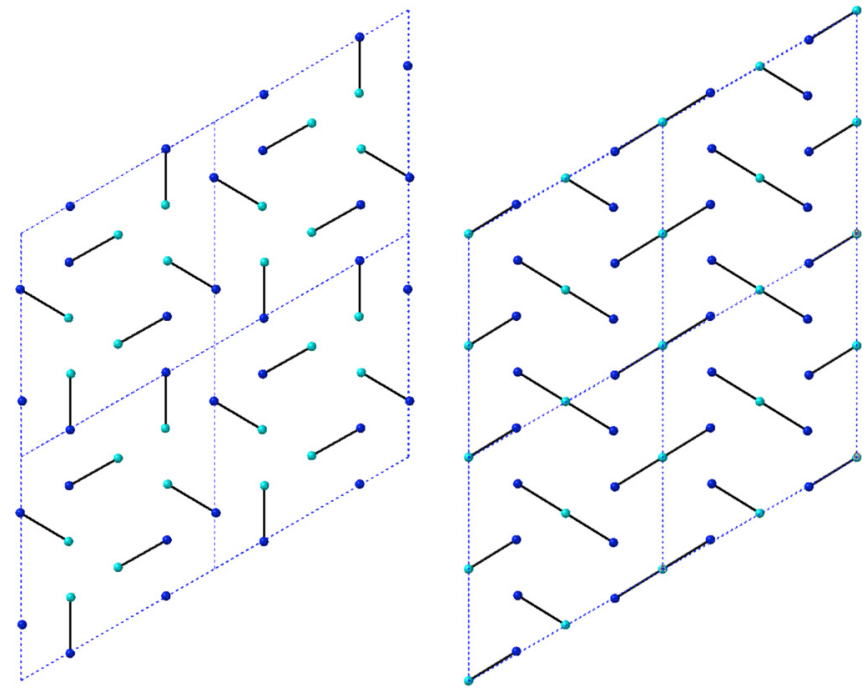

FIG. 3. Valence bond patterns for (left) the $\mathrm{R} \overline{3}$ phase (Znparatacamite) and (right) the $\mathrm{P} 2_{1} / \mathrm{n}$ phase (clinoatacamite). Similar patterns are seen for the $\mathrm{P}_{3} / \mathrm{m}$ and Pnma phases of barlowite, respectively. Only the copper ions are shown in a single kagome plane (the two crystallographically distinct sites are in blue and cyan).

This has previously been commented on in regards to the Pnma distortion seen in the closely related material, barlowite, $\mathrm{Cu}_{4}(\mathrm{OH})_{6} \mathrm{BrF}$ [24]. The distortion in the latter case is an $\mathrm{M}_{2}^{+}$mode due to the difference in stacking (AA), the highsymmetry phase being hexagonal $\left(\mathrm{P}_{3} / \mathrm{mmc}\right)$. In fact, there is a close analogy between the phase transitions seen in weakly Zn-doped clinoatacamite $\left(\mathrm{R} \overline{3} \mathrm{~m}\right.$ to $\mathrm{R} \overline{3}$ to $\left.\mathrm{P} 2_{1} / \mathrm{n}\right)$ and barlowite ( $\mathrm{P}_{3} / \mathrm{mmc}$ to $\mathrm{P}_{3} / \mathrm{m}$ to Pnma). In the former case, the primary distortion mode is $\mathrm{F}_{2}^{+}$with secondary modes $\mathrm{F}_{1}^{+}$and $\Gamma_{2}^{+}(\mathrm{R} \overline{3})$ and $\Gamma_{3}^{+}\left(\mathrm{P} 2_{1} / \mathrm{n}\right)$. For the latter, the primary distortion mode is $\mathrm{M}_{2}^{+}$with secondary modes $\mathrm{M}_{1}^{+}$and $\Gamma_{2}^{+}\left(\mathrm{P}_{3} / \mathrm{m}\right)$ and $\Gamma_{5}^{+}$ (Pnma). These differences again are due to $\mathrm{ABC}$ stacking (rhombohedral) versus AA stacking (hexagonal). This is summarized in Table I.

The detailed temperature dependence of these distortions has been considered by Malcherek et al. for clinoatacamite [25] and Welch et al. for Zn-paratacamite [9]. The resulting analysis from AMPLIMODES is shown in Fig. 4. Despite the expected first-order nature of the $R \overline{3}$ to $P 2_{1} / n$ phase transition, one sees that the $\mathrm{F}_{2}^{+}$distortion amplitude goes smoothly through the transition, and to a good approximation follows a Landau mean-field behavior of $\sqrt{T_{s 1}-T}$, where $T_{s 1}$ is the upper transition. Given the limited data, it is hard to quantify the $T$ dependence of the secondary modes. Nominally, the amplitude of the $\Gamma_{3}^{+}$mode should be quadratic in $\mathrm{F}_{2}^{+}$, but in reality it sets in discontinuously at $T_{s 2}$ (lower transition) due to the finite value of $\mathrm{F}_{2}^{+}$at $T_{s 2}$.

Returning to the valence bond patterns, a pinwheel pattern is also found in the higher-symmetry $\left(\mathrm{P}_{3} / \mathrm{m}\right)$ version of barlowite [24] (consistent with the above-discussed analogy with Zn-paratacamite) as also listed in Table I. The known list of patterns can be expanded by considering other materials in the class $\mathrm{A}_{2} \mathrm{Cu}_{3} \mathrm{BF}_{12}$ where $\mathrm{A}$ is an alkali metal and $\mathrm{B}$ a $4+$ cation [31]. These are also listed in Table I. In particular, 
TABLE I. Known valence bond solid (VBS) patterns in copper kagome materials. "Low $T$ " is the low-temperature crystal structure, and "High $T$ " the high-temperature one. "Mode" is the primary distortion mode. $z$ indicates the net buckling of the copper ions in the kagome plane along the hexagonal c axis (in $\AA$ ). The high- $T$ phase of $\mathrm{Cs}_{2} \mathrm{Cu}_{3} \mathrm{CeF}_{12}$ is unknown and so was determined from groupsubgroup relations. Note the large buckling often present in the copper fluorides as compared to the copper hydroxychlorides. References are clinoatacamite [25], paratacamite [9], barlowite [24], averievite [26], $\mathrm{Cs}_{2} \mathrm{Cu}_{3} \mathrm{CeF}_{12}$ [27], $\mathrm{Cs}_{2} \mathrm{Cu}_{3} \mathrm{ZrF}_{12}$ [28], $\mathrm{Cs}_{2} \mathrm{Cu}_{3} \mathrm{SnF}_{12}$ [29], and $\mathrm{Rb}_{2} \mathrm{Cu}_{3} \mathrm{SnF}_{12}$ [30].

\begin{tabular}{|c|c|c|c|c|c|}
\hline Low $T$ & High $T$ & VBS & Material & Mode & $z$ \\
\hline $\mathrm{P} 2_{1} / \mathrm{n}$ & $\mathrm{R} \overline{3} \mathrm{~m}$ & herringbone & clinoatacamite & $\mathrm{F}_{2}^{+}$ & 0.07 \\
\hline & & herringhone & $\mathrm{Cs}_{2} \mathrm{Cu}_{3} \mathrm{SnF}_{12}$ & $\mathbf{M}^{+}$ & 0.07 \\
\hline $\begin{array}{l}\mathrm{P} 2{ }_{1} / \mathrm{c} \\
\text { Pnma }\end{array}$ & P3m1 & herringbone & averievite & $\begin{array}{l}\mathrm{M}_{2}^{2} \\
\mathrm{M}^{+}\end{array}$ & $\begin{array}{l}0.00 \\
0.07\end{array}$ \\
\hline $\begin{array}{l}\text { Pnma } \\
\mathrm{R} \overline{3}\end{array}$ & $\mathrm{~Pb}_{3} / \mathrm{mmc}$ & herringbone & barlowite (1) & $\mathrm{M}_{2}^{+}$ & 0.07 \\
\hline R3 & $\mathrm{R} 3 \mathrm{~m}$ & pinwheel & $\begin{array}{l}\text { paratacamite } \\
\mathrm{Rb}_{2} \mathrm{Cu}_{3} \mathrm{SnF}_{12}\end{array}$ & $\mathrm{~F}_{2}^{+}$ & $\begin{array}{l}0.06 \\
0.38\end{array}$ \\
\hline $\mathrm{P}_{3} / \mathrm{m}$ & $\mathrm{P}_{3} / \mathrm{mmc}$ & pinwheel & barlowite (2) & $\mathrm{M}_{2}^{+}$ & 0.06 \\
\hline $\mathrm{P} 2{ }_{1} / \mathrm{m}$ & $\mathrm{R} \overline{3} \mathrm{~m}$ & zigzag & $\mathrm{Cs}_{2} \mathrm{Cu}_{3} \mathrm{ZrF}_{12}$ & $\mathrm{~F}_{2}^{-}$ & 0.73 \\
\hline Pnnm & $\mathrm{P}_{3} / \mathrm{mmc}$ & stripe & $\mathrm{Cs}_{2} \mathrm{Cu}_{3} \mathrm{CeF}_{12}$ & $\mathbf{M}_{3}^{+}$ & 3.93 \\
\hline
\end{tabular}

one also finds stripe phases $\left(\mathrm{Cs}_{2} \mathrm{Cu}_{3} \mathrm{CeF}_{12}\right)$ and zigzag phases $\left(\mathrm{Cs}_{2} \mathrm{Cu}_{3} \mathrm{ZrF}_{12}\right)$. In all cases in Table I, though, inversion symmetry is preserved.

This brings us to the SHG data on herbertsmithite [7]. They indicate a point group of either 2 or $\mathrm{m}$. A likely candidate, then, for the inversion-breaking space group is either $\mathrm{Cm}$ or C2. There are two ways this can happen. The first is by condensing a zone-centered polar mode $\left(\Gamma_{3}^{-}\right.$, Fig. 5). Possible valence bond patterns are shown in Fig. 6. Another way is by condensing an F-centered mode (Fig. 7) as shown in Fig. 8 (note, though, that the $\mathrm{F}_{2}^{-}$example given in Table I

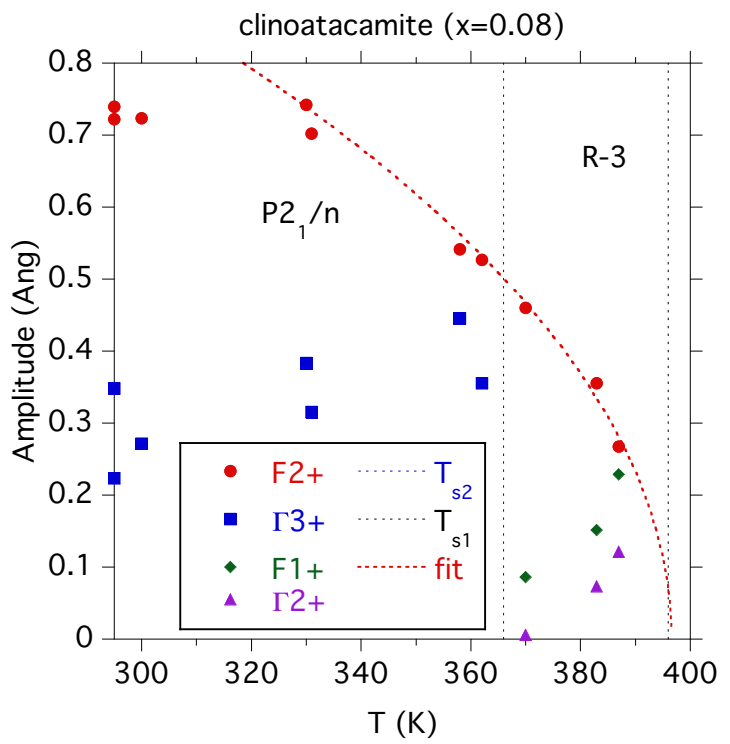

FIG. 4. Temperature dependence of the distortion mode amplitudes (in A) from clinoatacamite [25] generated by AMPLIMODES [11]. The red dashed curve is a Landau mean-field fit to the $F_{2}^{+}$mode.
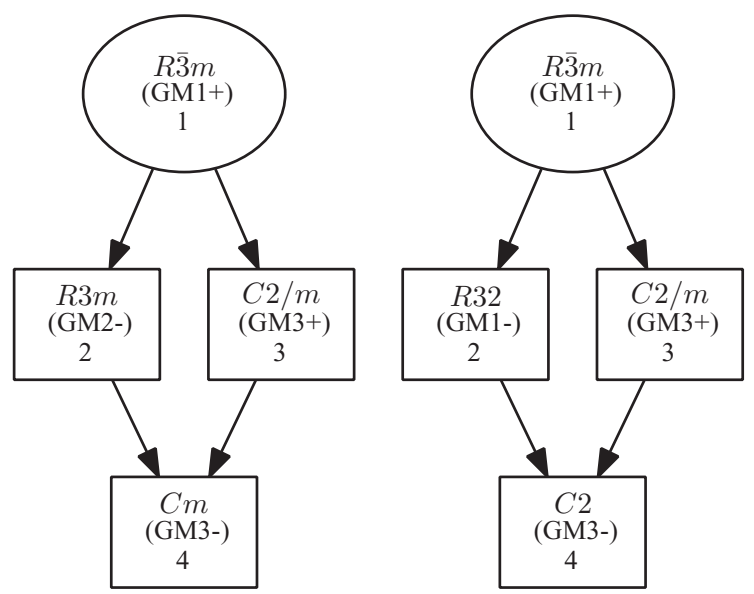

FIG. 5. Group-subgroup relation leading to (left) $\mathrm{Cm}$ and (right) $\mathrm{C} 2$, driven by a $\Gamma$-centered primary mode [13]. As indicated in these graphs, the primary mode is $\Gamma_{3}^{-}$, and the secondary modes, arising from the intermediate groups, are $\Gamma_{2}^{-}$and $\Gamma_{3}^{+}$for $\mathrm{Cm}$ and $\Gamma_{1}^{-}$and $\Gamma_{3}^{+}$ for $\mathrm{C} 2$.

preserves inversion). Again, these can take the form of stripes or zigzags, some of which result from buckled planes. Note that for illustrative purposes, these patterns are based on the shortest $\mathrm{Cu}-\mathrm{Cu}$ bonds. In reality, the strongest singlets will depend on the $\mathrm{Cu}-\mathrm{O}-\mathrm{Cu}$ bond angles, meaning oxygen atom displacements need to be considered once they are known. But one important difference to realize is that for the zonecentered case, one maintains an odd number of copper ions (per plane) in the unit cell. Therefore, we would anticipate an anisotropic spin liquid in this case rather than a valence bond solid [32]. For the zone-boundary modes, though, the unit cell size increases, resulting in an even number of copper ions instead, so this would be a valence bond solid.

One interesting point about the $\mathrm{Cm}$ and $\mathrm{C} 2$ space groups is that they are in general ferroelectric, with the polar axis

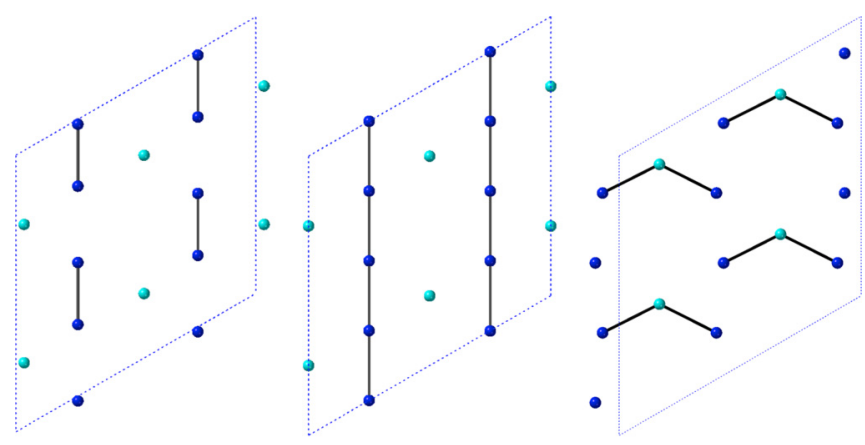

FIG. 6. Various $\mathrm{Cm}$ and $\mathrm{C} 2$ VBS patterns from a zone-centered mode $\left(\Gamma_{3}^{-}\right)$generated by ISODISTORT [12]. Left: $\mathrm{Cm} \mathrm{B}_{u 1}$ pattern; middle: $\mathrm{Cm} \mathrm{B}_{u 2}$ pattern; right: $\mathrm{C} 2 \mathrm{~A}_{u}$ pattern. Here $\mathrm{A}_{u}$ and $\mathrm{B}_{u}$ refer to point group symmetries of the copper kagome ions. The $\mathrm{Cm} \mathrm{A}_{u}$ pattern (not shown) is similar to the $\mathrm{Cm} \mathrm{B}_{u 1}$ one. These patterns are based on just copper-kagome-ion displacements and the shortest $\mathrm{Cu}$ $\mathrm{Cu}$ bonds (only the copper ions are shown in a single kagome plane; the two crystallographically distinct sites are in blue and cyan). The actual pattern will depend on the $\mathrm{Cu}-\mathrm{O}-\mathrm{Cu}$ bond angles once oxygenion displacements are known. 

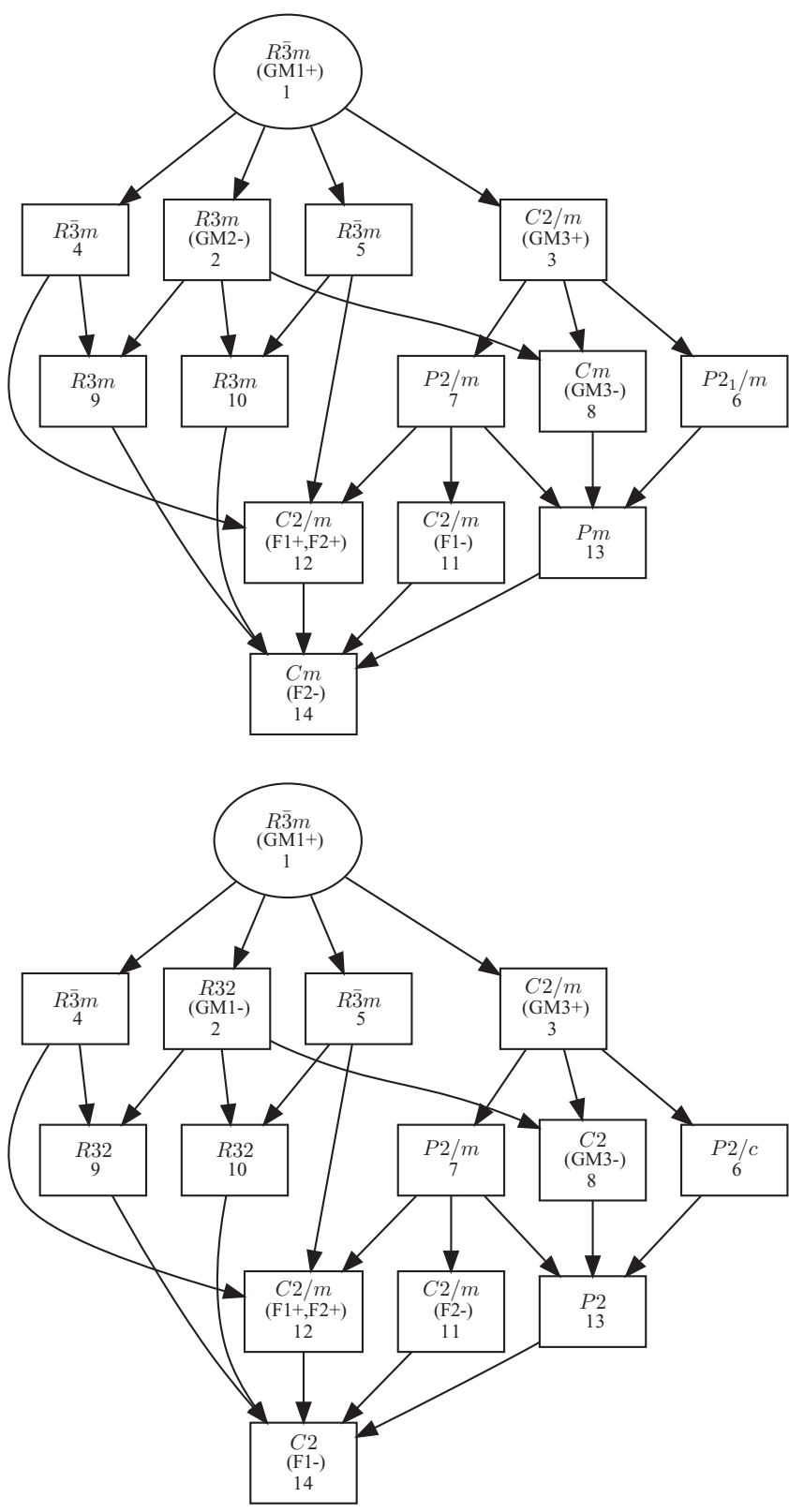

FIG. 7. Group-subgroup relation leading to (top) $\mathrm{Cm}$ and (bottom) C2, driven by an F-centered primary mode [13]. As indicated in these graphs, the primary mode is $\mathrm{F}_{2}^{-}$for $\mathrm{Cm}_{\text {and }} \mathrm{F}_{1}^{-}$for $\mathrm{C} 2$, with a variety secondary modes, arising from the intermediate groups, that differ for $\mathrm{Cm}$ and $\mathrm{C} 2$.

along the twofold (hexagonal $\mathrm{b}$ axis) for $\mathrm{C} 2$ and perpendicular to this axis for $\mathrm{Cm}$. Ferroelectric behavior has been claimed for the cobalt analog of clinoatacamite, with a proposed structural distortion of R3m [33]. Indications of ferroelectric-like behavior has also been seen in averievite [34], where a transition from the intermediate $\mathrm{P} 2{ }_{1} / \mathrm{c}$ phase to a lower-temperature phase of unknown symmetry has been observed [26]. This brings up the question of spin-lattice coupling. The SHG signal in herbertsmithite follows the predicted temperature dependence of the spin-spin correlator for a kagome lattice [7]. This is consistent with the temperature dependence of phonon linewidths [35]. Moreoever, the phonon frequen-
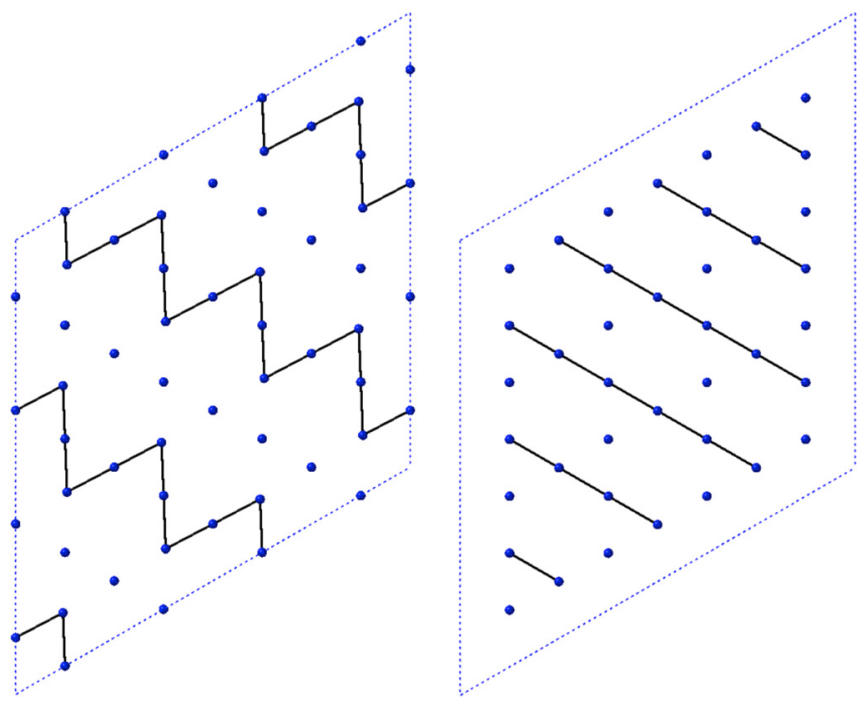

FIG. 8. Two of the Cm VBS patterns from a zone-boundary mode $\left(\mathrm{F}_{2}^{-}\right)$generated by ISODISTORT [12]. Left: One of the two $\mathrm{B}_{u 1}$ patterns; right: One of the two $\mathrm{B}_{u 2}$ patterns. The other two patterns are dimer patterns similar to Fig. 6(a). These patterns are based on just copper-kagome-ion displacements and the shortest $\mathrm{Cu}-\mathrm{Cu}$ bonds (only the copper ions are shown in a single kagome plane; there are seven crystallographically distinct sites). The actual pattern will depend on the $\mathrm{Cu}-\mathrm{O}-\mathrm{Cu}$ bond angles once oxygen-ion displacements are known.

cies shift [35], also indicative of spin-lattice coupling as has been studied extensively in pyrochlores [36,37]. The idea here is that the superexchange $J$ is sensitive to distortions given its dependence on the $\mathrm{Cu}-\mathrm{O}-\mathrm{Cu}$ bond angle, harking back to early work by Baltensperger [38], with the distortion occurring if the gain in exchange energy from increasing the bond angle exceeds the elastic cost of the lattice distortion. Spin-lattice couplings have been quantified in clinoatacamite using Raman data [39]. Ultimately, they can lead to multiferroic behavior, as observed in the distorted kagome material $\mathrm{KCu}_{3} \mathrm{As}_{2} \mathrm{O}_{7}(\mathrm{OH})_{3}$ [40].

Finally, what does all of this have to do with the KAHM? Density matrix renormalization group simulations have indicated that the ground state is a melted version of a 12-site diamond valence bond solid, closely related to the pinwheel pattern [41]. This has been further investigated by more recent numerical work [42], though related numerical simulations favor a Dirac spin liquid instead [43-45]. Small perturbations could certainly stabilize a valence bond solid [46-48], or an anisotropic spin liquid [32,49]. Given the above results, such models should be further explored to understand the rich physics of the Heisenberg model on a kagome lattice and its material realizations.

M.R.N. was supported by the Materials Sciences and Engineering Division, Basic Energy Sciences, Office of Science, U.S. Department of Energy. N.J.L. acknowledges partial support from an Institute for Quantum Information and Matter Postdoctoral Fellowship. D.H. acknowledges support from ARO PECASE Award No. W911NF-17-1-0204. 
[1] P. Lecheminant, B. Bernu, C. Lhuillier, L. Pierre, and P. Sindzingre, Phys. Rev. B 56, 2521 (1997).

[2] M. P. Shores, E. A. Nytko, B. M. Bartlett, and D. G. Nocera, J. Am. Chem. Soc. 127, 13462 (2005).

[3] P. Mendels and F. Bert, J. Phys. Soc. Jpn. 79, 011001 (2010).

[4] M. R. Norman, Rev. Mod. Phys. 88, 041002 (2016).

[5] T. H. Han, J. S. Helton, S. Chu, A. Prodi, D. K. Singh, C. Mazzoli, P. Muller, D. G. Nocera, and Y. S. Lee, Phys. Rev. B 83, 100402(R) (2011).

[6] A. Zorko, M. Herak, M. Gomilsek, J. van Tol, M. Velazquez, P. Khuntia, F. Bert, and P. Mendels, Phys. Rev. Lett. 118, 017202 (2017).

[7] N. J. Laurita, A. Ron, J. W. Han, A. Scheie, J. P. Sheckelton, R. W. Smaha, W. He, J.-J. Wen, J. S. Lee, Y. S. Lee, M. R. Norman, and D. Hsieh, arXiv:1910.13606.

[8] J. D. Grice, J. T. Szymanski, and J. L. Jambor, Can. Mineral. 34, 73 (1996).

[9] M. D. Welch, M. J. Sciberras, P. A. Williams, P. Leverett, J. Schluter, and T. Malcharek, Phys. Chem. Miner. 41, 33 (2014).

[10] A. R. Kampf, M. J. Sciberras, P. Leverett, P. A. Williams, T. Malcherek, J. Schluter, M. D. Welch, M. Dini, and A. A. M. Donoso, Mineral. Mag. 77, 3113 (2013).

[11] D. Orobengoa, C. Capillas, M. I. Aroyo, and J. M. Perez-Mato, J. Appl. Crystallogr. 42, 820 (2009).

[12] B. J. Campbell, H. T. Stokes, D. E. Tanner, and D. M. Hatch, J. Appl. Crystallogr. 39, 607 (2006).

[13] S. Ivantchev, E. Kroumova, G. Madariaga, J. M. Perez-Mato, and M. I. Aroyo, J. Appl. Crystallogr. 33, 1190 (2000).

[14] K. Momma and F. Izumi, J. Appl. Crystallogr. 44, 1272 (2011).

[15] A. K. Yadav, C. T. Nelson, S. L. Hsu, Z. Hong, J. D. Clarkson, C. M. Schlepuetz, A. R. Damodaran, P. Shafer, E. Arenholz, L. R. Dedon, D. Chen, A. Vishwanath, A. M. Minor, L. Q. Chen, J. F. Scott, L. W. Martin, and R. Ramesh, Nature 530, 198 (2016).

[16] V. M. Dubovik, L. A. Tosunyan, and V. V. Tugushev, Zh. Eksp. Teor. Fiz. 90, 590 (1986) [Sov. Phys. JETP 63, 344 (1986)].

[17] For simplicity, in this paper we focus on copper-ion displacements. Oxygen-ion displacements play an equally important role as indicated later. Which one dominates does not affect the definition of primary versus secondary modes, which is set by the group-subgroup relations and resulting Landau free energy, though the nature of the ion displacements depends critically on the symmetry of these modes.

[18] V. H. Crawford, H. W. Richardson, J. R. Wasson, D. J. Hodgson, and W. E. Hatfield, Inorg. Chem. 15, 2107 (1976). The linear relation of $J$ with $\mathrm{Cu}-\mathrm{O}-\mathrm{Cu}$ bond angle for copper hydroxides is such that the critical angle (where $J=0$ ) is $97.54^{\circ}$.

[19] K. Matan, T. Ono, Y. Fukumoto, T. J. Sato, J. Yamaura, M. Yano, K. Morita, and H. Tanaka, Nat. Phys. 6, 865 (2010).

[20] B.-J. Yang and Y. B. Kim, Phys. Rev. B 79, 224417 (2009).

[21] A. Wietek and A. M. Lauchli, arXiv:1908.02762.

[22] X. Rocquefelte, K. Schwarz, and P. Blaha, Sci. Rep. 2, 759 (2012).

[23] Y. Iqbal, H. O. Jeschke, J. Reuther, R. Valenti, I. I. Mazin, M. Greiter, and R. Thomale, Phys. Rev. B 92, 220404(R) (2015).
[24] R. W. Smaha, W. He, J. M. Jiang, C. J. Titus, J. Wen, Y.-F. Jiang, J. P. Sheckelton, S. G. Wang, Y.-S. Chen, S. J. Teat, A. A. Aczel, Y. Zhao, G. Xu, J. W. Lynn, H.-C. Jiang, and Y. S. Lee, arXiv:1907.00454.

[25] T. Malcherek, B. Mihailova, and M. D. Welch, Phys. Chem. Miner. 44, 307 (2017).

[26] A. S. Botana, H. Zheng, S. H. Lapidus, J. F. Mitchell, and M. R. Norman, Phys. Rev. B 98, 054421 (2018).

[27] T. Amemiya, M. Yano, K. Morita, I. Umegaki, T. Ono, H Tanaka, K. Fujii, and H. Uekusa, Phys. Rev. B 80, 100406(R) (2009).

[28] S. A. Reisinger, C. C. Tang, S. P. Thompson, F. D. Morrison, and P. Lightfoot, Chem. Mater. 23, 4234 (2011).

[29] L. J. Downie, C. Black, E. I. Ardashnikova, C. C. Tang, A. N. Vasiliev, A. N. Golovanov, P. S. Berdonosov, V. A. Dolgikh, and P. Lightfoot, CrystEngComm 16, 7419 (2014).

[30] L. J. Downie, S. P. Thompson, C. C. Tang, S. Parsons, and P. Lightfoot, CrystEngComm 15, 7426 (2013).

[31] K. Matan, T. Ono, G. Gitgeatpong, K. de Roos, P. Miao, S. Torii, T. Kamiyama, A. Miyata, A. Matsuo, K. Kindo, S. Takeyama, Y. Nambu, P. Piyawongwatthana, T. J. Sato, and H. Tanaka, Phys. Rev. B 99, 224404 (2019).

[32] B. K. Clark, J. M. Kinder, E. Neuscamman, Garnet Kin-Lic Chan, and M. J. Lawler, Phys. Rev. Lett. 111, 187205 (2013).

[33] X.-L. Xu, D.-D. Meng, X.-G. Zheng, I. Yamauchi, I. Watanabe, and Q.-X. Guo, Phys. Rev. B 95, 024111 (2017).

[34] T. Biesner, A. Pustogow, H. Zheng, J. F. Mitchell, and M. Dressel (unpublished).

[35] A. B. Sushkov, G. S. Jenkins, T.-H. Han, Y. S. Lee, and H. D. Drew, J. Phys.: Condens. Matter 29, 095802 (2017).

[36] Y. Yamashita and K. Ueda, Phys. Rev. Lett. 85, 4960 (2000).

[37] A. B. Sushkov, O. Tchernyshyov, W. Ratcliff II, S. W. Cheong, and H. D. Drew, Phys. Rev. Lett. 94, 137202 (2005).

[38] H. Baltensperger and J. S. Helman, Helv. Phys. Acta 41, 668 (1968).

[39] X.-D. Liu, X.-G. Zheng, D.-D. Meng, X.-L. Xu, and Q.-X. Guo, J. Phys.: Condens. Matter 25, 256003 (2013).

[40] G. J. Nilsen, Y. Okamoto, H. Ishikawa, V. Simonet, C. V. Colin, A. Cano, L. C. Chapon, T. Hansen, H. Mutka, and Z. Hiroi, Phys. Rev. B 89, 140412(R) (2014).

[41] S. Yan, D. A. Huse, and S. R. White, Science 332, 1173 (2011).

[42] A. Ralko, F. Mila, and I. Rousochatzakis, Phys. Rev. B 97, 104401 (2018).

[43] Y. Ran, M. Hermele, P. A. Lee, and X.-G. Wen, Phys. Rev. Lett. 98, 117205 (2007).

[44] Y. Iqbal, D. Poilblanc, and F. Becca, Phys. Rev. B 89, 020407(R) (2014).

[45] Y.-C. He, M. P. Zalatel, M. Oshikawa, and F. Pollmann, Phys. Rev. X 7, 031020(R) (2017).

[46] Y. Huh, M. Punk, and S. Sachdev, Phys. Rev. B 84, 094419 (2011).

[47] Y. Iqbal, F. Becca, and D. Poilblanc, New J. Phys. 14, 115031 (2012).

[48] K. Hwang, Y. Huh, and Y. B. Kim, Phys. Rev. B 92, 205131 (2015).

[49] C. Repellin, Y.-C. He, and F. Pollmann, Phys. Rev. B 96, 205124 (2017). 